\title{
CORRIGENDUM TO "AN INCREMENTAL ALGORITHM FOR CONSTRUCTING SHORTEST WATCHMAN ROUTES"
}

\author{
XUEHOU TAN \\ School of High-Technology for Human Welfare, Tokai University \\ 317 Nishino, Numazu 410-0321, Japan \\ E-mail: tan@fc.u-tokai.ac.jp \\ TOMIO HIRATA and YASUYOSHI INAGAKI \\ Faculty of Engineering, Nagoya University \\ Chikusa-ku, Nagoya 464-8603, Japan \\ Received 28 May 1998 \\ Revised 12 April 1999 \\ Communicated by D. T. Lee
}

\begin{abstract}
This corrigendum fixes an error that appears in the previously published papers concerning the watchman route problem. A modification to our incremental watchman route algorithm previously presented in this journal is made, which gives an $O\left(n^{4}\right)$ time solution to the watchman route problem.
\end{abstract}

Keywords: Algorithms, watchman routes, adjustments, dynamic programming.

\section{Introduction}

An error in Lemma 5 of our paper ${ }^{3}$ was recently pointed out. To fix it, we need to modify the whole algorithm somewhat, and also change the $O\left(n^{3}\right)$ time bound claimed in Ref. [3] to $O\left(n^{4}\right)$. The reader should refer to the original paper for problem statement and definitions.

Let us first give a brief description of previous watchman route algorithms and the error found by Hammar and Nilsson. ${ }^{2}$ In 1991, Chin and Ntafos ${ }^{1}$ claimed an $O\left(n^{4}\right)$ time solution to the watchman route problem for simple polygons that have a starting point $s$ on their boundaries (i.e., watchman routes pass through $s$ ). Their algorithm first finds an initial watchman route and then computes the shortest watchman route by repeatedly adjusting the current one with the adjust at the first choice rule. That is, the adjustment on the cut of the smallest index is first done. In the proofs of Lemma 8 and Theorem 2 of Ref. [1], Chin and Ntafos made an observation that the adjustments on any previous cut resulting from an adjustment on a cut $C_{j}$ should be all in the same direction. It then follows that no adjustment, before selecting an adjustment on $C_{k}$ with $k>j$, occurs more than once at a cut 
intersection (see the proof of Theorem 2 in Ref. [1]). We made a similar observation that no adjusting directions can be changed in the process of making a one-placeadjustable route non-adjustable (see Lemma 5 of Ref. [3]). It is then shown in Lemma 6 of Ref. [3] that the number of adjustments required is linear, which leads to an $O\left(n^{3}\right)$ time incremental algorithm. Later, a quadratic time algorithm is also given using a divide-and-conquer strategy. ${ }^{4}$

Very recently, Hammar and Nilsson pointed out that the observations made in Refs. $[1,3]$ were not generally true and the adjustments on the cuts of lower indices would change their directions because of a $(+1)$ - or $(-1)$-adjustment. ${ }^{2}$ That is, $(+1) /(-1)$-adjustments may cause the routes, obtained by the "adjust at the first choice" rule, to oscillate forth and back on the cuts of lower indices. (Note that the oscillation on a cut refers to the situation where routes reflect forth and back on different fragments of the cut, rather than within the interior of a single fragment.) Hammar and Nilsson tried to fix the error by using a different choice rule, however, their algorithm also suffers from the same problem (e.g, a (+1)-adjustment on the cut, along which the adjustable reflection point $r$ moves in their $\mathrm{AN}$-algorithm, ${ }^{2}$ requires changing the directions of the adjustments early made on the cuts of lower or higher indices). Since the adjusting rules given in the previous work ${ }^{1,2,3}$ only check the local optimality of the current route, a (+1)- or (-1)-adjustment requires changing the directions of adjustments performed before. It turns out that no polynomial time bounds for the previous algorithms are guaranteed.

In this corrigendum, we fix the error stated above by introducing a dynamic programming approach to our incremental algorithm. ${ }^{3}$ Specifically, we compute the shortest partial watchman routes from $s$ to all intersections and endpoints of cuts, which helps us give an incremental algorithm that adjusts a route only when it is one-place-adjustable. Our modified algorithm runs in $O\left(n^{4}\right)$ time. (The error of Chin and Ntafos' algorithm can be fixed in the same way, and the time bound is $O\left(n^{5}\right)$ by their timing analysis. ${ }^{1}$ On the other hand, the divide-and-conquer algorithms given in Refs. [2,4] cannot be used to improve the $O\left(n^{4}\right)$ time bound established in this corrigendum.)

\section{The Incremental Watchman Route Algorithm Modified}

A route from $s$ to a point on cut $C_{i}$ is a partial watchman route to a point on $C_{i}$ (or a watchman path as denoted in Ref. [3]) if it lies in the essential piece of $C_{i}$ and visits all cuts $C_{1}, C_{2}, \ldots, C_{i-1}$. To simplify the presentation, we will consider cut endpoints as cut intersections and also the starting point $s$ itself as an additional cut $C_{m+1}$, where $m$ denotes the number of essential cuts.

Our modified incremental algorithm can be summarized as follows.

\section{Algorithm: WatchmanRoute.}

1. Find all essential cuts $C_{1}, C_{2}, \ldots, C_{m}, C_{m+1}(=s)$, indexed in the order of a clockwise scan of the polygon boundary starting at the given point $s$, and then compute cut intersections among $C_{1}, C_{2}, \ldots, C_{m}$. 


\section{FOR $i=1$ TO $m+1$ DO}

Compute the shortest partial watchman routes from $s$ to all intersections on $C_{i}$ using the previously obtained partial watchman routes.

3. Report the final route from $s$ to $C_{m+1}(=s)$ as the shortest watchman route.

Since the time complexity of WatchmanRoute is dominated by Step 2, we will show how it can be done in $O\left(n^{4}\right)$ time. Let $p_{i, j}$ denote the intersection of $C_{i}$ with $C_{j}$, and let $p_{i, \text { left }}$ and $p_{i, \text { right }}$ denote the left and right endpoints of $C_{i}$ (as viewed from $s$ ), respectively. First, the shortest paths in the given polygon $P$ from $s$ to the intersections on $C_{1}$ are the shortest partial watchman routes to the intersections on $C_{1}$. Assume now that we have computed the shortest partial watchman routes to all intersections on $C_{1}, C_{2}, \ldots, C_{i-1}$. To compute the shortest partial watchman route to an intersection $p_{i, j}$ on $C_{i}$, we first find an initial one-place-adjustable route $R_{i, j}^{0}$. Consider the situation that $C_{i}$ does not intersect with $C_{i-1}$. Let $R_{i-1, \text { right }}$ denote the shortest partial watchman route to the endpoint $p_{i-1, \text { right }}$ of $C_{i-1}$. Then the route $R_{i, j}^{0}$ is formed by combining $R_{i-1, r i g h t}$ with the shortest path between $p_{i-1, r i g h t}$ and $p_{i, j}$ (Fig. 1a). Since route $R_{i-1, r i g h t}$ is defined within the essential piece of $C_{i-1}$, route $R_{i-1, \text { right }}$ may overlap with $C_{i-1}$. See Fig. $1 \mathrm{~b}$ for an example. Consider now the situation that $C_{i}$ intersects with $C_{i-1}$. If $p_{i, j}$ does not dominate $C_{i-1}$, then route $R_{i, j}^{0}$ is formed by combining the shortest partial watchman route to $p_{i-1, i}$ with the line segment $\left(p_{i-1, i}, p_{i, j}\right)$. See Figs. 1 c-d. If $p_{i, j}$ dominates $C_{i-1}$, then the route $R_{i, j}^{0}$ can be obtained by replacing the portion of the shortest partial watchman route to $p_{i-1, i}$, which starts at the last reflection point and ends at $p_{i-1, i}$, with the shortest path from the last reflection point to $p_{i, j}$. See Figs. 1e-f.

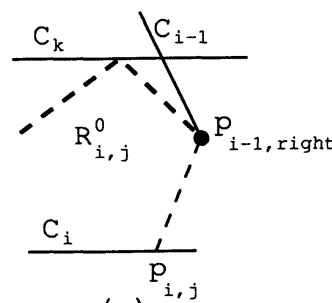

(a)

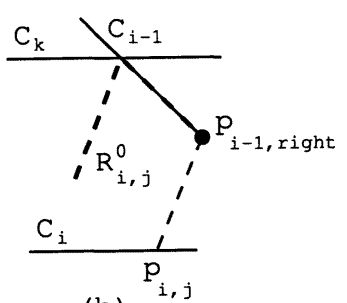

(b)

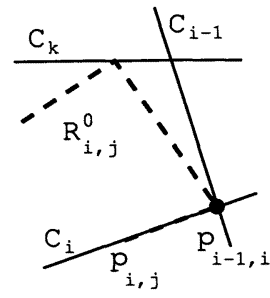

(c)

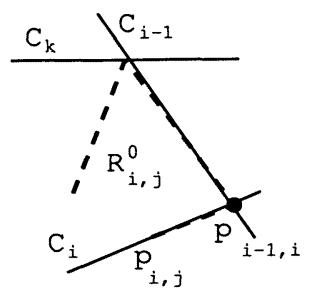

(d)

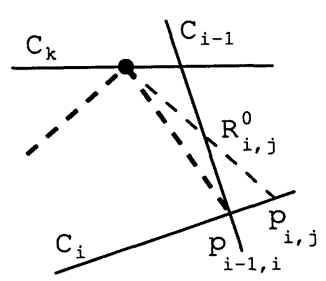

(e)

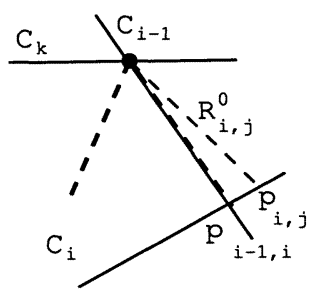

(f)

Fig. 1. Possible configurations between $R_{i, j}^{0}$ and $C_{i-1}$. 
Observe that the incoming angle of route $R_{i, j}^{0}$ with the cut $C_{k}(k<i-1)$ in Fig. $1 \mathrm{~b}$ and Fig. $1 \mathrm{~d}$ has to be greater than the outgoing angle, and no adjustment occurs on $C_{k}$ until the overlap of the current route with $C_{i-1}$ is removed. Therefore, the obtained route $R_{i, j}^{0}$ is one-place-adjustable (the place is shown by a dot in Fig. 1).

After the route $R_{i, j}^{0}$ is found, we perform the only adjustment to $R_{i, j}^{0}$. Let $R_{i, j}^{1}$ denote the resulting route, which is computed by the rolling out method described in Refs. [1,3]. Generally, route $R_{i, j}^{1}$ may be adjustable on several cuts. We do not perform any adjustment to $R_{i, j}^{1}$. Instead, we take out the shortest partial watchman route to the intersection point on the cut $C_{l}$, where the last adjustment (i.e., index $l$ is the biggest among the adjustable cuts) to $R_{i, j}^{1}$ is possible, and concatenate it to the part of $R_{i, j}^{1}$ from the intersection on $C_{l}$ to $p_{i, j}$. Let $R_{i, j}^{2}$ denote the resulting route. Note that route $R_{i, j}^{2}$ lies in the essential piece of $C_{l}$. If $R_{i, j}^{2}$ is non-adjustable, then it is the shortest partial watchman route to $p_{i, j}$ and we are done. Otherwise, it is adjustable only on $C_{l}$, and the adjustment can be performed. In this way, we can eventually find the shortest partial watchman route to $p_{i, j}$.

We now show that the oscillation does not occur in our adjusting process.

Lemma 1 Let $R_{i, j}^{k}$ denote a one-place-adjustable route, which is adjustable at a point $p$ on a cut $C_{h}$. Assume that the adjustment requires the reflection point of the next route $R_{i, j}^{k+1}$ on $C_{h}$ to move to the left (right) of $p$. Then any subsequent one-place-adjustable route $R_{i, j}^{l}(l \geq k+2)$, in the rest of the process of computing the shortest partial watchman route to $p_{i, j}$, cannot be adjustable on $C_{h}$ at $p$ again (i.e., there is no oscillation on $C_{h}$ ).

Proof. We prove it by contradiction. Assume that there is a one-placeadjustable route $R_{i, j}^{l}(l \geq k+2)$ that is adjustable on $C_{h}$ at $p$. Since the length of $R_{i, j}^{l}$ is shorter than that of $R_{i, j}^{k}$, route $R_{i, j}^{l}$ has to differ from route $R_{i, j}^{k}$. Observe that each portion of the one-place-adjustable routes $R_{i, j}^{k}$ and $R_{i, j}^{l}$ from $s$ to $p$ and from $p$ to $p_{i, j}$ is a shortest partial watchman route. Thus, either the shortest partial watchman route from $s$ to $p$ of cut $C_{h}$ in route $R_{i, j}^{k}$ differs from that in route $R_{i, j}^{l}$ or the shortest partial watchman route from $p$ of cut $C_{h}$ to $p_{i, j}$ in route $R_{i, j}^{k}$ differs from that in route $R_{i, j}^{l}$. It contradicts with the uniqueness of the shortest partial watchman route ${ }^{a}$.

The following lemma is essentially the same as Lemma 6 in Ref. [3]. For completeness, we restate it and give below a simple proof.

Lemma 2 The shortest partial watchman route to an intersection point $p_{i, j}$ on a cut $C_{i}$ can be found in $O(m n)$ time, where $m$ is the number of essential cuts, provided that the shortest partial watchman routes to all intersections on the previous cuts are known.

Proof. We first claim that in the process of computing the shortest partial watchman route to $p_{i, j}$, once an active cut becomes inactive it can never be active again. Suppose that cut $C_{h}$ becomes inactive, because of a (-1)-adjustment on a cut $C_{k}$. It follows from Lemma 1 that the active fragment of $C_{k}$ dominates $C_{h}$ since then. If $C_{k}$ becomes inactive too, because of a (-1)-adjustment on another cut $C_{k^{\prime}}$,

\footnotetext{
${ }^{a}$ The uniqueness of the shortest watchman route is shown by Chin and Ntafos. ${ }^{1}$ We can easily modify their proof for the shortest partial watchman route.
} 
then the active fragment of $C_{k^{\prime}}$ dominates $C_{k}$ and $C_{h}$. Since polygon $P$ is simple, the claim is proved by a simple induction on the sequence of $(-1)$-adjustments.

Since a (-1)-adjustment makes an active cut inactive, it follows from the claim that there are at most $i-1(-1)$-adjustments in the process of computing the shortest partial watchman route to $p_{i, j}$. (Note that all cuts $C_{i+1}, C_{i+2}, \ldots, C_{m}$ need not be active in computing the shortest partial watchman route to $p_{i, j}$. Thus, any cut $C_{k}$ with $k>i$ can only participate in 0-adjustments.) Since a $(+1)$ adjustment makes an inactive cut active, the number of $(+1)$-adjustments required is bounded the number of the cuts that can be active, which is $O(i)$ again. For 0 -adjustments, let us charge the cost of a 0 -adjustment to the participating inactive cut. Since the intersection of essential pieces of active cuts forms several disjoint convex chains within polygon $P$, an inactive cut can intersect with at most two active cuts. It then follows from Lemma 1 that an inactive cut participates in at most two 0-adjustments. Thus, the total number of 0-adjustments needed is $O(m)$. As the time required to perform an adjustment or take out a shortest route is $O(n)$, the proof is completed.

Since the total number of cut intersections is $O\left(n^{2}\right)$, we have the following result.

Theorem 1 Given a simple polygon $P$ of $n$ vertices and a starting point $s$ on the boundary of $P$, it is possible to find the shortest watchman route through $s$ within polygon $P$ in $O\left(n^{4}\right)$ time.

Note in addition. Although the time bounds of the previous algorithms $1,2,3,4$ remain to be analyzed further, these algorithms may work much faster in most cases. Since an adjustment produces a shorter route, the oscillation seldom occurs in practice.

\section{References}

1. W.P. Chin and S. Ntafos, Shortest watchman routes in simple polygons, Disc. Comp. Geom. 6 (1991) 9-31.

2. M. Hammar and B.J. Nilsson, Concerning the time bounds of existing shortest watchman route algorithms, in Proc. FCT'97, LNCS 1279 (1997) 210-221.

3. X. Tan, T. Hirata and Y. Inagaki, An incremental algorithm for constructing shortest watchman routes, Int. J. Comp. Geom. \& Appl. 3 (1993) 351-365.

4. X. Tan and T. Hirata, Constructing shortest watchman routes by divide-andconquer, in Proc. ISAAC'93, LNCS 762 (1993) 68-77. 